\title{
Das unendlich lange Trennrohr mit Endbehälter
}

\author{
Von Alfred Klemm \\ Aus dem Kaiser-Wilhelm-Institut für Chemie, Tailfingen \\ (Z. Naturforschg. 3 a, 211-216 [1948]; eingegangen am 1. April 1948)
}

\begin{abstract}
Es wird der raum-zeitliche Konzentrationsverlauf eines seltenen Isotops in einem unendlich langen Trennrohr mit durchmischtem Endbehälter unter Verwendung des Quellenintegrals berechnet. Die Erstreckung der Anreicherung ins Trennrohr hinein ist $1,77 \sqrt{D^{\prime} t}$ für $l \ll \sqrt{D^{\prime} t}$ und $1,50 \sqrt{D^{\prime} t}$ für $l \gg \sqrt{D^{\prime} t}$. Die theoretische Plattenzahl (Vervielfachung) ist $2 w \sqrt{t} / \sqrt{\pi D^{\prime}}$ für $l \ll \sqrt{D^{\prime} t}$ und $w t / l$ für $l \gg \sqrt{D^{\prime} t}$. Dabei ist $w$ die effektive Geschwindigkeit des Gegenstroms, $D^{\prime}$ die effektive Diffusionskonstante, $l$ die äquivalente Länge des Endbehälters und $t$ die Zeit. Die Größen $w$ und $D^{\prime}$ werden für den Fall des Thermodiffusionsverfahrens, des chemischen Austauschverfahrens und des elektrolytischen Überführungsverfahrens interpretiert.
\end{abstract}

$\mathrm{O}$ bwohl eine vorgegebene An- oder Abreicherung eines Isotops mit einem Trennrohr (gleichgültig, ob es sich um Thermodiffusion, elektrolytische Überführung oder sonst ein Trennprinzip handelt) am raschesten erreicht wird, wenn das Trennrohr an dem betreffenden Ende geschlossen ist, also keinen Endbehälter besitzt, werden doch aus apparativen Gründen, und weil die Einstellzeit bei Dauerbetrieb keine Rolle spielt, oft Trennapparaturen mit Endbehälter gebaut. Den Einstellvorgang eines ThermodiffusionsTrennrohres endlicher Länge, welches am einen Ende geschlossen ist und am anderen Ende einen Endbehälter trägt, hat B a r d e e $\mathrm{n}^{1}$ berechnet. Der durch Laplace-Transformation gewonnene Konzentrationsverlauf wird dort in einer Reihendarstellung gegeben, die um so besser konvergiert, je näher man der Endeinstellung ist. Bei Trennrohren für kondensierte Stoffe wird aber wegen der gegenüber Gasen etwa $10^{5}$-mal kleineren Diffusionskonstante die Endeinstellung u. U. keineswegs erreicht, und es ist dann zweckmäßig, die Rechnung für den Idealfall eines unendlich langen Trennrohres durchzuführen, wobei die Integration in geschlossener Form möglich ist. Die Rechnungen für endliche und unendliche Trennrohre ohne Endbehälter findet man übrigens im Prinzip schon bei Mas on und Weaver ${ }^{2}$.

Wir wollen unser Problem zunächst folgendermaßen formulieren:

Gesucht ist die über den Rohrquerschnitt ge-

1 J. B a r d e en, Physic. Rev. 57, 35 [1940].

2 M. Mason u. W. We aver, Physic. Rev. 23, 424 [1924]. mittelte Konzentration $c(z, t)$, welche der Differentialgleichung

$$
\frac{\partial c}{\partial t}=-v \frac{\partial c}{\partial z}+D^{\prime} \frac{\partial^{2} c}{\partial z^{2}}
$$

mit der Anfangsbedingung

$$
c=c_{0} \quad \text { für } t=0 \text { und } 0<z<\infty
$$

und der Randbedingung

$$
v c-D^{\prime} \frac{\partial c}{\partial z}+l \frac{\partial c}{\partial t}=0
$$

$$
\text { für } z=0 \text { und } 0<t<\infty
$$

genügt. Hier ist $z$ die Ortskoordinate in Richtung des Trennrohres und $t$ die Zeit. $v$ ist die über den Rohrquerschnitt gemittelte, in $z$-Richtung und relativ zur Rohrwand gerechnete Geschwindigkeit, die das betrachtete Isotop durch das angewandte Trennprinzip erhält. $D^{\prime}$ ist eine effektive Diffusionskonstante, die den über den Rohrquerschnitt gemittelten Konzentrationsausgleich beschreibt, indem bei ausgeschalteter Trennwirkung der Teilchenstrom durch $-D^{\prime} \partial c / \partial z$ gegeben ist. $v$ und $D^{\prime}$ sind räumlich und zeitlich konstant angenommen. Der Endbehälter, in dem die Konzentration z. B. durch Rühren räumlich konstant gehalten wird, habe den gleichen Querschnitt wie das Trennrohr und die Länge $l$. Er befinde sich links der Ebene $z=0$, während das Trennrohr rechts dieser Ebene liegt.

Die so formulierte Randwertaufgabe trifft nur für ein seltenes Isotop in Strenge zu.

Durch Einführung der dimensionslosen Größen 


$$
\zeta=z v / L^{\prime}, \quad \tau=t v^{2} / D^{\prime}, \quad \lambda=l v / D^{\prime}
$$

und der neuen, der Gleichung

$$
c=c_{0} \exp (\zeta / 2-\tau / 4) f(\zeta, \tau)
$$

genügenden Variablen $f$ reduziert sich das Problem auf die Aufgabe, die Funktion $f(\zeta, \tau)$ zu suchen, welche der Differentialgleichung

$$
\frac{\partial f}{\partial \tau}=\frac{\partial^{2} t}{\partial \xi^{2}}
$$

mit der Anfangsbedingung

$$
f=\exp (-\zeta / 2) \text { für } \tau=0 \text { und } 0<\zeta<\infty
$$

und der Randbedingung

$$
\frac{2-\lambda}{4} f-\frac{\partial f}{\partial \zeta}+\lambda \frac{\partial f}{\partial \tau}=0
$$

genügt.

$$
\text { für } \zeta=0 \text { und } 0<\tau<\infty
$$

Der Quellenintegralansatz

$f=\frac{1}{2 \sqrt{\pi \tau}} \int_{0}^{\infty} W(a) \exp \left[-\left(\frac{a-\zeta}{2 \sqrt{\tau}}\right)^{2}\right] d a$ Randbedingung zu erfüllen. Für positive $\alpha$ wird $W(\alpha)$ durch Einsetzen von (7) in (9) bestimmt:

$$
W(\alpha)=\exp (-a / 2) \text { für } 0<a<\infty .
$$

genügt (6) und enthält in der beliebigen Funktion $W(\alpha)$ die Möglichkeit, die Anfangs- und

(8) erfordert

$$
\begin{array}{r}
\int_{-\infty}^{+\infty} W(a)\left(\frac{2-\lambda}{4}-\frac{a}{2 \tau}-\frac{\lambda}{2 \tau}+\frac{\lambda \alpha^{2}}{4 \tau^{2}}\right) \\
\cdot \exp \left[-\left(\frac{a}{2 \sqrt{\tau}}\right)^{2}\right] d a=0 .
\end{array}
$$

Mit Benützung der Beziehung

$$
-\frac{a}{2 \tau} \exp \left[-\left(\frac{a}{2 \sqrt{\tau}}\right)^{2}\right]=\frac{\partial}{\partial a} \exp \left[-\left(\frac{a}{2 \sqrt{\tau}}\right)^{2}\right]
$$

wird (11) durch partielle Integrationen umgewandelt in

$$
\begin{array}{r}
\int_{-\infty}^{+\infty}\left[\frac{2-\lambda}{4} W(a)-\frac{d W(a)}{d a}+\lambda \frac{d^{2} W(a)}{d a^{2}}\right] \\
\cdot \exp \left[-\left(\frac{a}{2 \sqrt{\bar{\tau}}}\right)^{2}\right] d a=0 .
\end{array}
$$

(13) kann nur für alle $\tau$ erfüllt sein, wenn die erste eckige Klammer in (13) für positives und negatives $\alpha$ den umgekehrt gleichen Wert besitzt, und wenn sie für $\alpha=0$ endlich ist, wenn also gilt

$$
\begin{gathered}
\frac{2-\lambda}{4} W(-a)+\frac{d W(-a)}{d a}+\lambda \frac{a^{2} W(-a)}{d a^{2}} \\
+\exp \left(-\frac{a}{2}\right)=0 \quad \text { für } 0<a<\infty \\
W(-a)=1 \quad \text { für } a=0 \\
\frac{d W(-a)}{d a}=\frac{1}{2} \quad \text { für } a=0 .
\end{gathered}
$$

Die Lösung des Gleichungssystemes (14), (15), (16) lautet

$$
W(-a)=\frac{1}{\lambda-1} a \exp \left(-\frac{a}{2}\right)+\frac{1}{(\lambda-1)^{2}} \exp \left(-\frac{a}{2}\right)+\frac{\lambda^{2}-2 \lambda}{(\lambda-1)^{2}} \exp \left(-\frac{2-\lambda}{2 \lambda} a\right)
$$

(10) und (17) in (9) eingesetzt ergibt

$$
\begin{aligned}
f=\frac{1}{2 \sqrt{\pi \tau}} \int_{0}^{\infty}\left\{\exp \left[-\frac{a}{2}-\left(\frac{a-\zeta}{2 \sqrt{\tau}}\right)^{2}\right]\right. & +\left[\frac{1}{\lambda-1} a \exp \left(-\frac{a}{2}\right)+\frac{1}{(\lambda-1)^{2}} \exp \left(-\frac{a}{2}\right)\right. \\
& \left.\left.+\frac{\lambda^{2}-2 \lambda}{(\lambda-1)^{2}} \exp \left(-\frac{2-\lambda}{2 \lambda} a\right)\right] \exp \left[-\left(\frac{a+\zeta}{2 \sqrt{\tau}}\right)^{2}\right]\right\} d a .
\end{aligned}
$$

Unter Verwendung der Beziehung

$$
\frac{1}{\sqrt{\pi \tau}} \int_{0}^{\infty} \exp \left[-r a-\left(\frac{a+\zeta}{2 \sqrt{\tau}}\right)^{2}\right] d a=\exp \left(r^{2} \tau+r \zeta\right)\left[1-\Phi\left(\frac{2 r \tau+\dot{\zeta}}{2 \sqrt{\tau}}\right)\right],
$$


führen wir in (18) das Fehlerintegral

$$
\Phi(x)=\frac{2}{\sqrt{\pi}} \int_{0}^{x} \exp (-\alpha) \alpha^{2} \alpha
$$

ein und erhalten, wenn wir noch $f$ in (5) einsetzen:

$$
c=c_{0} \Theta(\zeta, \tau, \lambda) .
$$

$$
\Theta=\frac{1}{2}\left\{1-\Phi\left(\frac{\tau-\zeta}{2 \sqrt{\tau}}\right)+\frac{1}{1-\lambda}\left(\frac{1}{1-\lambda}+\tau+\zeta\right) \exp (\zeta)\left[1-\Phi\left(\frac{\tau+\zeta}{2 \sqrt{\tau}}\right)\right]\right.
$$

$$
\left.+\frac{\lambda^{2}-2 \lambda}{(1-\lambda)^{2}} \exp \left(\frac{1-\lambda}{\lambda^{2}} \tau+\frac{1}{\lambda} \zeta\right)\left[1-\Phi\left(\frac{\frac{2-\lambda}{\lambda} \tau+\zeta}{2 \sqrt{\tau}}\right)\right]-\frac{1}{1-\lambda} \frac{2}{\sqrt{\pi}} \sqrt{\tau} \exp \left[-\left(\frac{\tau-\zeta}{2 \sqrt{\tau}}\right)^{2}\right]\right\} \text {. }
$$

Für $\lambda=0$ geht (22) in den Ausdruck von $\mathrm{M}$ a s o n und $\mathrm{W}$ e a v e $\mathrm{r}^{2}$ über.

Wir wollen nun unser Resultat speziell auf die Isotopentrennung anwenden, unterscheiden deshalb zwei Isotope $i$ und $k$ durch Indices links oben und führen den Trennfaktor ${ }^{i k} Q$ ein durch

Dann ist

$$
{ }^{i k} Q=\frac{{ }^{i}{ }^{k} c_{0}}{{ }^{k} c^{i} c_{0}} .
$$

$$
\ln { }^{i k} Q=\ln \Theta\left({ }^{i} \zeta,{ }^{i} \tau,{ }^{i} \lambda\right)-\ln \Theta\left({ }^{k} \zeta,{ }^{k} \tau,{ }^{k} \lambda\right) .
$$

Wegen der Ähnlichkeit der Isotope entwickeln wir (24) nach Potenzen von ${ }^{i} D^{\prime}-{ }^{k} D^{\prime}$ und ${ }^{i} v-{ }^{k} v$ und berücksichtigen nur die erste Näherung:

$$
\begin{aligned}
\ln ^{i k} Q & =\left(\frac{\partial \ln \Theta}{\partial \zeta} \frac{\partial \zeta}{\partial D^{\prime}}+\frac{\partial \ln \Theta}{\partial \tau} \frac{\partial \tau}{\partial D^{\prime}}\right. \\
\left.+\frac{\partial \ln \Theta}{\partial \lambda} \frac{\partial \lambda}{\partial D^{\prime}}\right)_{v=0}\left({ }^{i} D^{\prime}-{ }^{k} D^{\prime}\right)+\left(\frac{\partial \ln \Theta}{\partial \zeta} \frac{\partial \zeta}{\partial v}\right. & \\
+ & \left.\frac{\partial \ln \Theta}{\partial \tau} \frac{\partial \tau}{\partial v}+\frac{\partial \ln \Theta}{\partial \lambda} \frac{\partial \lambda}{\partial v}\right)_{v=0}\left({ }^{i} v-{ }^{k} v\right) .
\end{aligned}
$$

Nun ist für $v=0$ :

$$
\Theta=1, \frac{\partial \dot{s}}{\partial D^{\prime}}=\frac{\partial \tau}{\partial D^{\prime}}=\frac{\partial \lambda}{\partial D^{\prime}}=\frac{\partial \tau}{\partial v}=0 .
$$

Also ist

$$
\ln ^{i k} Q=\left(\frac{\partial \Theta}{\partial \zeta} \frac{z}{D^{\prime}}+\frac{\partial \Theta}{\partial \lambda} \cdot \frac{l}{D^{\prime}}\right)_{v=0}\left({ }^{i} v-{ }^{k} v\right) .
$$

Nach Ausführung der Differentiationen in (27) erhält man folgendes Ergebnis ${ }^{3}$ :

$$
\begin{gathered}
\ln ^{i k} Q=-f^{i k} a \\
{ }^{i k} a=\left({ }^{i} v-{ }^{k} v\right) / w, \\
f=\frac{2}{\sqrt{\pi}} \sqrt{\tau^{\prime}} \Psi\left(\frac{\zeta^{\prime}}{\sqrt{\tau^{\prime}}}, \frac{\lambda^{\prime}}{\sqrt{\tau^{\prime}}}\right), \\
\Psi\left(\frac{\zeta^{\prime}}{\sqrt{\tau^{\prime}}} \frac{\lambda^{\prime}}{\sqrt{\tau^{\prime}}}\right)=l v / D^{\prime}, \tau^{\prime}=t w^{2} / D^{\prime}, \lambda^{\prime}=l w / D^{\prime}, \\
=\left\{1+\frac{\sqrt{\pi}}{2} \frac{\lambda^{\prime}}{\sqrt{\tau^{\prime}}}\left[1-\Phi\left(\frac{\zeta}{2 \sqrt{\tau^{\prime}}}+\frac{\sqrt{\tau^{\prime}}}{\lambda^{\prime}}\right)\right]\right. \\
\left.\exp \left[\left(\frac{\zeta}{2 \sqrt{\tau^{\prime}}}+\frac{\sqrt{\tau^{\prime}}}{\lambda^{\prime}}\right)^{2}\right]\right\} \exp \left[-\left(\frac{\zeta^{\prime}}{2 \sqrt{\tau^{\prime}}}\right)^{2}\right] \\
-\frac{\sqrt{\pi}}{2}\left(\frac{\zeta^{\prime}+\lambda^{\prime}}{\sqrt{\tau^{\prime}}}\right)\left[1-\Phi\left(\frac{\zeta}{2 \sqrt{\tau^{\prime}}}\right)\right] .
\end{gathered}
$$

Durch die Gleichungen (30), (31), (32) ist der raumzeitliche Verlauf der Größe $f$, die man ,Vervielfachung“ oder auch ,theoretische Plattenzahl" nennen mag, gegeben. Bevor wir hierauf eingehen, wollen wir aber die Bedeutung der Größe ${ }^{i k} \alpha$, der ,effektiven Geschwindigkeit des Gegenstromes" $w$ und der ,effektiven Diffusionskonstante" $D^{\prime}$ an Hand von einigen Beispielen erörtern.

Beim Clusius-Dickel-Verfahren setzen wir

$$
{ }^{i k} a=a \frac{\Delta T}{T} .
$$

${ }^{3}$ Für $l=0$ vgl. A. K l e m m, Z. Naturforschg. 1. $252[1946]$. 
Durch Vergleich z. B. mit B ardee n s ${ }^{1}$ Formeln für ebene Wände folgt dann

$$
\begin{aligned}
w & =\frac{d^{2} \varrho g}{720 \eta} \frac{\Delta T}{T}, \\
D^{\prime} & =D+1,43 \frac{w^{2} d^{2}}{D} .
\end{aligned}
$$

Es bedeuten: $\alpha=$ Thermodiffusionsfaktor, $d=\mathrm{Ab}$ stand der Wände, $\varrho=$ Dichte, $g=$ Erdbeschleunigung, $\eta=$ Zähigkeit, $D=$ Diffusionskonstante, $\Delta T=$ Temperaturdifferenz zwischen den Wänden. In (35) stammt das zweite Glied rechts von der Rückmischung durch Querdiffusion, die durch die Kombination von Gegenstrom und Konzentrationsgradienten entsteht, während das erste Glied die gewöhnliche Längsdiffusion erfaßt.

Wenn unsere Rechnung für Urey-Kolonnen angewendet werden soll, hat man unseren Größen $i{ }^{k} \boldsymbol{\alpha}, w$ und $D^{\prime} \mathrm{z}$. B. folgende Bedeutung zu geben ${ }^{4}$ :

$$
\begin{gathered}
{ }^{i k_{C}}=\ln \frac{{ }^{i} h_{1}^{*}{ }^{k} h_{z}^{*}}{{ }^{k} h_{1}^{*}{ }^{i} h_{2}^{*}}, \\
w=\frac{l_{1}}{h_{1}+h_{2}}, \\
I^{\prime}=w^{2} \frac{h_{1}+h_{2}}{k^{\prime} h_{1} h_{2}} .
\end{gathered}
$$

Die Größen $h(\mathrm{Mol} / \mathrm{cm})$ und $l(\mathrm{Mol} / \mathrm{sec})$ sind Substanzmengen pro Säulenlängeneinheit und Transporte in Säulenrichtung.

$$
h_{n}={ }^{i} h_{n}+{ }^{k} h_{n}, l_{1}={ }^{i} l_{1}+{ }^{k} l_{1}=l_{2} .
$$

Linke obere Indices kennzeichnen Isotope, rechte untere Phasen (z. B. 1=flüssig, 2=gasförmig), Sterne rechts oben bedeuten thermodynamisches Gleichgewicht. $k^{\prime}(\mathrm{cm} / \mathrm{sec})$ ist die summarische Reaktionsgeschwindigkeit, definiert durch die Gleichung

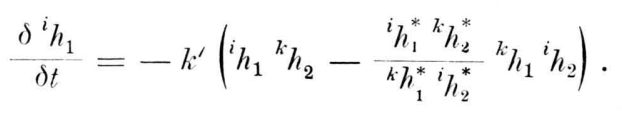

Der Operator $\delta / \delta t$ bezeichnet eine zeitliche Änderung allein auf Grund des Austausches senkrecht zur Rohrachse. Die Längsdiffusion trägt wenig zu $D^{\prime}$ bei und ist deshalb in (38) weggelassen.

${ }^{4}$ Vgl. z. B. K. Cohen, J. Chem. Physics 8, 588 [1940].

5 Vgl. a. A. K l e m m, Z. Naturforschg. 3 b, 62 [1948].
Im Falle der Isotopenanreicherung durch elektrolytische Überführung setzen wir

$$
{ }^{i k_{c}}=\ln \frac{{ }^{i} w}{{ }^{k} w},
$$

wb ${ }^{i} w$ und ${ }^{k} w$ die Translationsgeschwindigkeiten der Isotope relativ zu einem geeigneten Bezugsystem sind. Das Bezugsystem ist ,geeignet", wenn ${ }^{i k} \alpha$ unabhängig von der elektrischen Feldstärke wird. Für verdünnte Lösungen ist das Lösungsmittel, für geschmolzene Salze der Schwerpunkt der Gegenionen als Bezugsystem geeignet ${ }^{5}$.

$w$ kann hier mit ${ }^{i} w$ oder ${ }^{k} w$ gleichgesetzt werden. Nach Westhave ${ }^{6}$ ist, wenn die Ionenwanderung durch eine Gegenströmung des gesamten Elektrolyten im Mittel kompensiert wird, die effektive Diffusionskonstante $D^{\prime}$ wiederum die Summe der gewöhnlichen Diffusionskonstante und eines von der Konvektion herrührenden Gliedes. Zum Beispiel gilt für den Fall, daß die Gegenströmung eine gewöhnliche Poiseuillesche Strömung in einem Rohr vom Radius $r$ ist:

$$
D^{\prime}=D+0,02 \frac{u^{2} r^{2}}{D} \text {. }
$$

Wir kommen nun zur Diskussion der durch (30), (31), (32) gegebenen sogen. Vervielfachung $f$, die die Wirksamkeit der Apparatur charakterisiert.

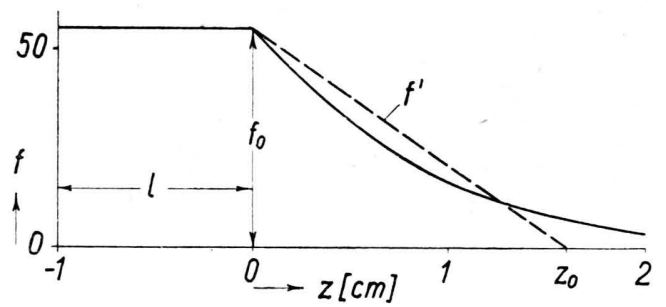

Abb. 1. Der räumliche Verlauf der Vervielfachung $f$ nach Gln. (30), (31), (32) für den Fall $l=1 \mathrm{~cm}$, $w=10^{-2} \mathrm{~cm} / \mathrm{sec}, D^{\prime}=10^{-4} \mathrm{~cm}^{2} / \mathrm{sec}, t=10^{4} \mathrm{sec}$. Die lineare Ersatzfunktion $f^{\prime}$ (gestrichelt) erfüllt die Beziehung $\int_{0}^{z_{0}} f^{\prime} d z=\int_{0}^{\infty} f d z$ (Gl. 47).

In Abb. 1 ist zunächst an einem Beispiel der räumliche Verlauf von $f$ gezeigt. Links von $z=0$ ist der $1 \mathrm{~cm}$ lange Endbehälter zu denken, in dem wegen des Rührens $f$ konstant ist, rechts von $z=0$ ist das Trennrohr mit kontinuierlich gegen 0 gehender Vervielfachung.

6 J. W. We st h a v e r, J. Res. Nat. Bur. Standards 38, 169 [1947]. 


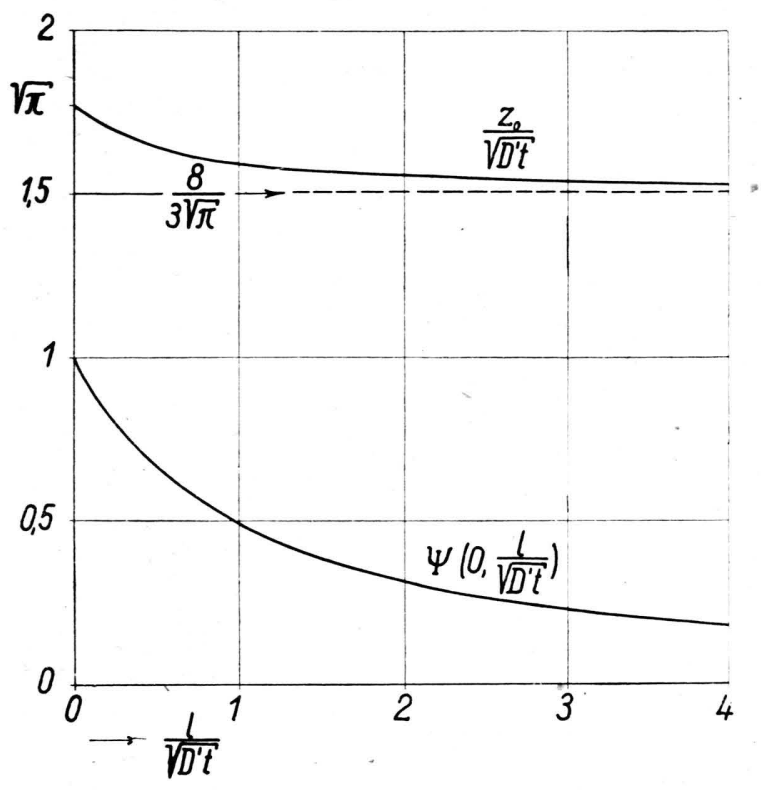

Abb. 2. Die Funktion $\psi\left(0, l / \sqrt{D^{\prime} t}\right)$ (Gl. 43) und die Erstreckung $z_{0}$ (Gl.53) der Konzentrationsverschiebungen im Trennrohr in Abhängigkeit von $t, D^{\prime}$ und $l$.
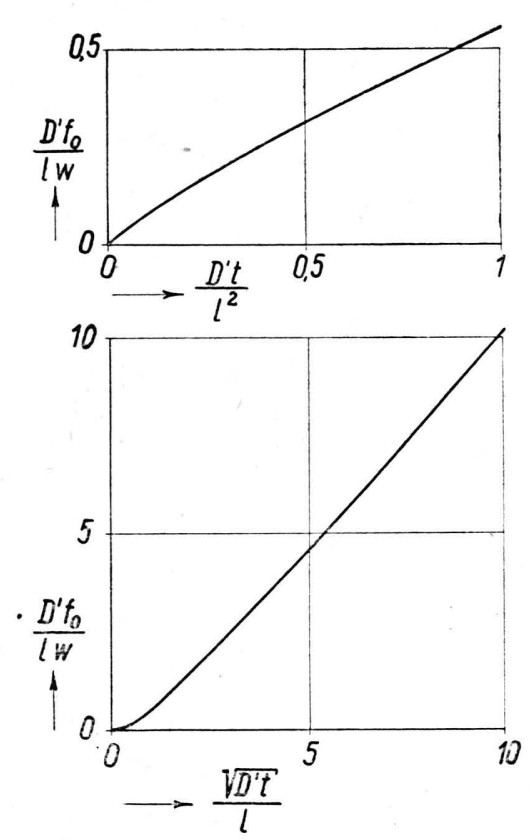

Abb. 3. Die Vervielfachung im Endbehälter $f_{0}$ in $\mathrm{Ab}$ hängigkeit von $t, w, D^{\prime}$ und $l$ nach Gl. 42 .

Die Vervielfachung $f_{0}$ im Endbehälter ergibt sich durch Nullsetzen von $\zeta$ in (32) zu

$$
f_{0}=\frac{2}{\sqrt{\pi}} \sqrt{\tau^{\prime}} \Psi\left(0, \frac{\hat{\imath}^{\prime}}{\sqrt{\tau^{\prime}}}\right)
$$

$$
\begin{aligned}
& \Psi^{\prime}\left(0, \frac{\lambda^{\prime}}{\sqrt{\tau^{\prime}}}\right)= \\
& 1-\frac{\sqrt{\pi}}{2} \frac{\hat{\iota}^{\prime}}{\sqrt{\tau^{\prime}}}\left\{1-\left[1-\Phi\left(\frac{\sqrt{\tau^{\prime}}}{\hat{\iota}^{\prime}}\right)\right] \exp \left[\left(\frac{\sqrt{\overline{\tau^{\prime}}}}{\lambda^{\prime}}\right)^{2}\right]\right\} .
\end{aligned}
$$

Den Verlauf von $\psi\left(0, \lambda^{\prime} / \sqrt{\tau^{\prime}}\right)$ zeigt Abb. 2, und den zeitlichen Anstieg von $f_{0}$ für beliebige $w, D^{\prime}$ und $l$ Abb. 3. Für $l \ll \sqrt{D^{\prime} t}$ ergibt sich

$$
f_{0}=\frac{2}{\sqrt{\pi}} \sqrt{\tau^{\prime}}=\frac{2 w \sqrt{t}}{\sqrt{\pi D^{\prime}}},
$$

und fuir $l \gg \sqrt{D^{\prime} t}$ hat man

$$
f_{0}=\frac{2}{1 \pi} \sqrt{\tau^{\prime}} \cdot \frac{\sqrt{x}}{2} \frac{\sqrt{\tau^{\prime}}}{\lambda}=\frac{u t}{l} .
$$

Wir betrachten nun noch den räumlichen Verlauf von $f$ im Trennrohr. Für die Diskussion ist es praktisch, die Funktion $f$ durch eine lineare Funktion $f^{\prime} \mathrm{zu}$ ersetzen (s. Abb. 1), die den Bedingungen

$f^{\prime}=f \quad$ für $z=0, \quad f^{\prime}=0 \quad$ für $z=z_{0}$,

wo

$$
\int_{0}^{\infty} f d z=\int_{0}^{z^{\prime \prime}} f^{\prime} d z
$$

genügt. $z_{0}$ wäre dann etwa als ,Erstreckung der. Anreicherung" zu bezeichnen. Um $z_{0}$ zu berechnen, schreiben wir die leichtverständliche Gleichung

$$
\int_{-l}^{\infty}\left(c_{0}-c\right) d z=c_{0} v t
$$

für die Isotope $i$ und $k$ an, dividieren beziehungsweise durch ${ }^{i} c_{0}$ und ${ }^{k} c_{0}$ und subtrahieren die Gleichungen voneinander. Dann ergibt sich, wenn man noch näherungsweise

setzt, mit (29)

$$
\frac{{ }^{i} c}{{ }^{i} c_{0}}-\frac{{ }^{k} c}{{ }^{k} c_{0}}=\ln { }^{i k} Q
$$

$$
\int_{-l}^{\infty} \ln { }^{i k} Q d z=-{ }^{i k} \alpha v t,
$$

oder mit (28)

$$
f_{0} l+\int_{0}^{\infty} f d z=\imath t .
$$




\section{Wegen}

folgt mit (42)

$$
\int_{0}^{\infty} f d z=\frac{1}{2} f_{0} z_{0}
$$

$$
z_{0}=\frac{\sqrt{\pi D^{\prime} t}}{\Psi\left(0, l / \sqrt{D^{\prime} t}\right)}-2 l .
$$

$z_{0}$ in Abhängigkeit von $t, D^{\prime}$ und $l$ ist in Abb. 2 dargestellt. Man erkennt, daß die Erstreckung der Anreicherung $z_{0}$ nur wenig von $l$ abhängt und im wesentlichen proportional zu $\sqrt{D^{\prime}} \mathrm{t}$ ist. Der Proportionalitätsfaktor ist $\sqrt{\pi}=1,77$ für $l \ll \sqrt{D^{\prime} t}$ und $8 /(3 \sqrt{\pi})=1,50$ für $l \gg \sqrt{D^{\prime} t}$.

Zum Schluß sei daran erinnert, daß bei der Endeinstellung eines Trennrohres der Länge $Z$ ohne Entnahme gilt

$$
f=Z w / D^{\prime} .
$$

Die Größe

$$
h=D^{\prime} / w
$$

nennt man den ,theoretischen Plattenabstand“. Wollte man eine entsprechende Größe $h$ für den Einstellvorgang des unendlich langen Trennrohres einführen, so hätte man etwa zu setzen

$$
h=z_{0} / f_{0} .
$$

Für den Fall, daß kein Endbehälter vorhanden ist, ergäbe sich dann

$$
h=\frac{\pi}{2} \frac{D^{\prime}}{w},
$$

und mit wachsendem Endbehälter würde $h$ beliebig groß werden.

Anm. b. d. Korr.: In J. Res. Nat. Bur. Standards Washington 39, 397-409 [1947] ist die Arbeit .Calculations on counter-current electromigration " von G. B r e it und F. L. F r i ed $\mathrm{m}$ a $\mathrm{n} n$ erschienen, in der meine Gl. (22) unter Verzicht auf die Herleitung vorweggenommen ist. Außerdem wird dort der Fall des Trennrohres endlicher Länge mit Endbehälter am einen und konstanter Konzentration am anderen Ende behandelt.

\title{
Widerstandsänderung einer Platinfolie durch Gasbelađung
}

\author{
Von Werner BraunbeK ${ }^{1}$ \\ (Z. Naturforschg. 3 a, 216-220 [1948]; eingegangen am 6. April 1948)
}

\begin{abstract}
Eine freitragende Platinfolie von knapp $2 \mu$ Dicke zeigt in einer Gasatmosphäre eine Erniedrigung ihres elektrischen Widerstandes um einige 10-4 gegenüber dem Vakuumwert. Der Effekt ist von Gas zu Gas verschieden. Bei den drei untersuchten Gasen ist er bei Sauerstoff am größten, bei Argon etwas, bei Helium sehr viel geringer. Nach Abpumpen des Gases geht der Widerstand nahezu wieder auf den alten Wert hinauf. Die Widerstandserniedrigung entspricht einer zusätzlichen Metallschicht von einer-je rach Art des Gases verschiedenen Dicke, welche jedoch immer in der Größenordnung einer Atomschicht liegt. Der Effekt kann gedeutet werden als Freisetzung von Leitungselektronen der Oberflächen-Metallatome durch die Gasbeladung, welche im Vakuum infolge der Oberflächen-Deformation des Potentials in ihrer Beweglichkeit behindert sind.
\end{abstract}

$\mathrm{S}_{\mathrm{i}}^{\mathrm{e}}$ eit langem ist bekannt, daß sich der elektrische Widerstand dünner Metallfolien von der Oberfläche her beeinflussen läßt. Die Leitfähigkeits-Anomalien dünner Metallschichten, die auf eine nichtleitende Unterlage aufgedampft (oder durch Kathodenzerstäubung aufgebracht) werden, werden zu einem wesentlichen Teil dem Einfluß der Untèrlage zugeschrieben. Insbeson-

1 Universität Tübingen. Die Versuche wurden 1939 im Physikalischen Institut durchgeführt; die Ergebnisse können aber aus kriegsbedingten Gründen erst jetzt veröffentlicht werden. dere zeigen aber die zahlreichen Versuche ${ }^{2}$, durch ein statisches elektrisches Feld an der Oberfläche einer Metallschicht deren elektrischen Widerstand zu beeinflussen, trotz der starken Widersprüche

2 H. A. P e r kin s, C. R. hebd. Séances Acad. Sci. 127, 303 [1923]; Physic. Rev. 25, 584 [1925]. - M. P i e r u c c i, Nuovo Cimento 8, 193, 198 [1931]; 9, 33 [1932]; 11, 277 [1934].-G. Polvani, Nuovo Cimento 8, 198 [1931]; 9, 69 [1932]. - R. D e a g l i o, Nuovo Cimento 11, 288 [1934]. - E. P e r ucca, Atti R. Accad. Sci. Torino [Classe Sci. fisich. mat. nat.] $\mathbf{6 9}$, 166 [1934]. - A. D e ubne r, Naturwiss. 22, 239 [1934]; Ann. Physik (5) 20,449 [1934]. 